\title{
Elementos Fundamentales que Componen la Radio Cognitiva y Asignación de Bandas Espectrales
}

\author{
Danilo A. López ${ }^{(1) *}$, Edwin R. Trujillo ${ }^{(1)}$ y Oscar E. Gualdron ${ }^{(2)}$ \\ (1) Universidad Distrital Francisco José de Caldas, Carrera 7 No. 40 - 53 Bogotá-Colombia. \\ (e-mail:dalopezs@udistrital.edu.co,erivas@udistrital.edu.co) \\ (2) Universidad de Pamplona, Campus Universitario, Pamplona-Colombia, \\ (e-mail: oscar.gualdron@unipamplona.edu.co)
}

* Autor a quien debe ser dirigida la correspondencia.

Recibido Jun. 11, 2014; Aceptado Ago. 8, 2014; Versión final recibida Sep. 9, 2014

\begin{abstract}
Resumen
Se describen los elementos fundamentales que componen la radio cognitiva y se analiza la asignación de bandas espectrales. Dentro de los principales problemas en la inserción de nuevas aplicaciones y tecnologías inalámbricas, está la falta de espectro radioeléctrico para su asignación, debido a la manera ineficiente de distribución del espectro radioeléctrico disponible, que es actualmente asignado de forma estática. La radio cognitiva nace como un método que propone una solución a la problemática, gestionando dinámicamente el recurso. Una de las etapas que integran esta tecnología es la decisión de espectro, en la que se selecciona y asigna las bandas frecuenciales a partir de los requisitos de calidad de servicio de los usuarios cognitivos. Este artículo plantea una propuesta para el desarrollo de un proyecto de investigación que optimice el proceso de selección de canales reduciendo el tiempo de asignación para elevar su rendimiento.
\end{abstract}

Palabras clave: radio cognitiva, toma de decisiones espectrales, asignación estática, asignación dinámica

\section{Basic Elements that Compose Cognitive Radio and Mapping Spectral Bands}

\begin{abstract}
Fundamental elements of the cognitive radio and assignment of spectral bands are described and analyzed. Among the main problems in the insertion of new applications and wireless technologies is the lack of radio spectrum for allocation due to the inefficient distribution of available radio spectrum, which is statically allocated at present. The cognitive radio paradigm was born as a method for proposing a solution to the problem, dynamically managing the resource. One of the stages that make up this technology is the decision of the spectrum that selects and allocates frequency bands based on quality of service requirements of the cognitive users. This article presents a proposal for the development of a research project that optimizes the selection of channel allocation by reducing the time to improve their performance.
\end{abstract}

Keywords: cognitive radio, spectral making decisions, static allocation, dynamic allocation 


\section{INTRODUCCIÓN}

Actualmente existen algunas regiones con licencia donde las frecuencias en realidad son parcialmente usadas causando degradación de la calidad de servicio en algunas regiones espectrales (bandas celulares) por falta de disponibilidad, mientras que en otras se subutilice como en las bandas VHF/UHF (FCC, 2002; Wellens, et al., 2012; McHenry, 2005; McHenry, et al., 2006; López, et al., 2009; Matinmikko, et al., 2010; Wellens, et al., 2010; Taher, et al., 2011), como consecuencia de la utilización inadecuada y esporádica, variables que asociadas a la demanda de espectro.

Surge el acceso dinámico al espectro (DSA) como solución a la problemática existente. (Mitola, 1992) expone que la administración de las bandas electromagnéticas se puede realizar de forma dinámica a través de la radio cognitiva (CR) Las características fundamentales de CR están cimentadas en la capacidad cognitiva y reconfigurabilidad autónoma (Park, 2007) e incluyen 4 funciones principales que son en su orden monitorización, decisión, compartición y movilidad del espectro (figura 3), siendo uno de los menos investigados la toma de decisiones (Masonta, et al., 2013). Cabe destacar que en (Pla, et al., 2010) se argumenta que la función de decisión puede ser vista como un problema de optimización y un reto para la CR, donde el objetivo es seleccionar una banda no utilizada que maximice el rendimiento de la aplicación y el nodo cognitivo (SU), sin causar interferencia a los usuarios licenciados (PUs) a partir de detección espectral, condiciones existentes en el medio ambiente y exigencias de los usuarios.

En toma de decisiones, la mayor parte de las investigaciones se centran en plantear soluciones de tipo cualitativo (Wang, 2011; Gutiérrez, et al., 2013; Husain et al., 2013; Canberk, et al., 2011; Khabazian, et al., 2012; Barnes y Maharaj, 2013; Motani y Liang, 2013; Suzan y Fatih, 2012; Derakhshani y Le-Ngoc, 2012), existiendo carencia de propuestas cuantitativas que validen los resultados; enfocándose estas en la caracterización del usuario primario o licenciado (PU) y relegando el usuario secundario o cognitivo (SU) (Masonta, et al., 2013); variable que debiera tenerse en cuenta ya que redunda en un mejor uso y distribución del canal; en la selección del espectro, la mayoría de investigaciones como (Barnes y Maharaj, 2013; Motani y Liang, 2013; Suzan y Fatih, 2012; Derakhshani y Le-Ngoc, 2012; Brah, et al., 2012; WonYeol y Akyildiz, 2008; Akyildiz, 2011) han inclinado sus modelos a la utilización de estrategias reactivas (selección de la banda de transmisión una vez el usuario solicite el servicio) con un gran inconveniente, debido al tiempo (variable crítica dentro del contexto de las telecomunicaciones) que se consume en la búsqueda y selección de la frecuencia libre para el SU.

La implementación de una estrategia proactiva (donde la identificación del canal se decide segundos antes a la llegada del usuario cognitivo), permitiría disminuir los tiempos, mejorando la etapa de decisión de espectro, en consecuencia optimizando el rendimiento del sistema cognitivo. De acuerdo a ello se plantea: ¿Cómo se podría reducir el tiempo de asignación de bandas espectrales a los SUs sin degradar la comunicación del PU y haciendo más equitativo el uso del canal?. La solución a la pregunta de investigación inicia por contextualizar la propuesta fundamentándola en la situación actual, incluyendo aspectos relevantes abordados por la comunidad científica en la caracterización de los PUs, SUs, canales o medios de transmisión y los métodos de selección de bandas espectrales; para posteriormente presentar y sustentar la idea de investigación que se pretende desarrollar.

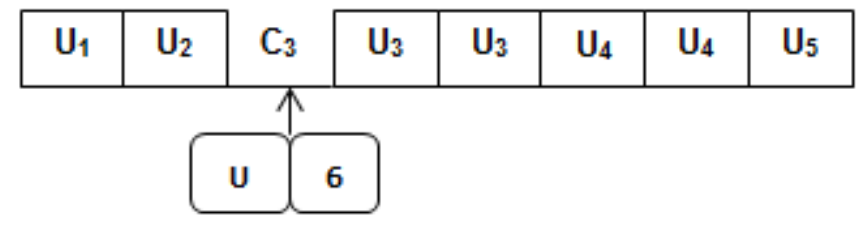

Fig. 1: Escacez de bandas para el transporte de los datos (Bolivar, 2012).

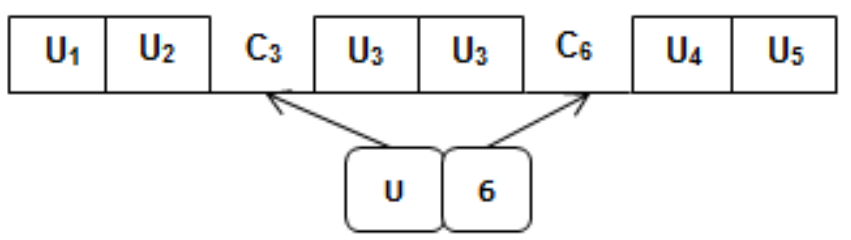

Fig. 2: Uso no óptimo del espectro (Bolivar, 2012). 


\section{CONCEPTUALIZACION DE LA PROPUESTA}

En este apartado se presenta la conceptualización general de la propuesta de asignación de bandas espectrales basado en la situación actual. La propuesta se centra en la etapa de decisión espectral (figura 3), la cual puede ser distribuida (Ad-Hoc) o centralizada (basada en infraestructura).

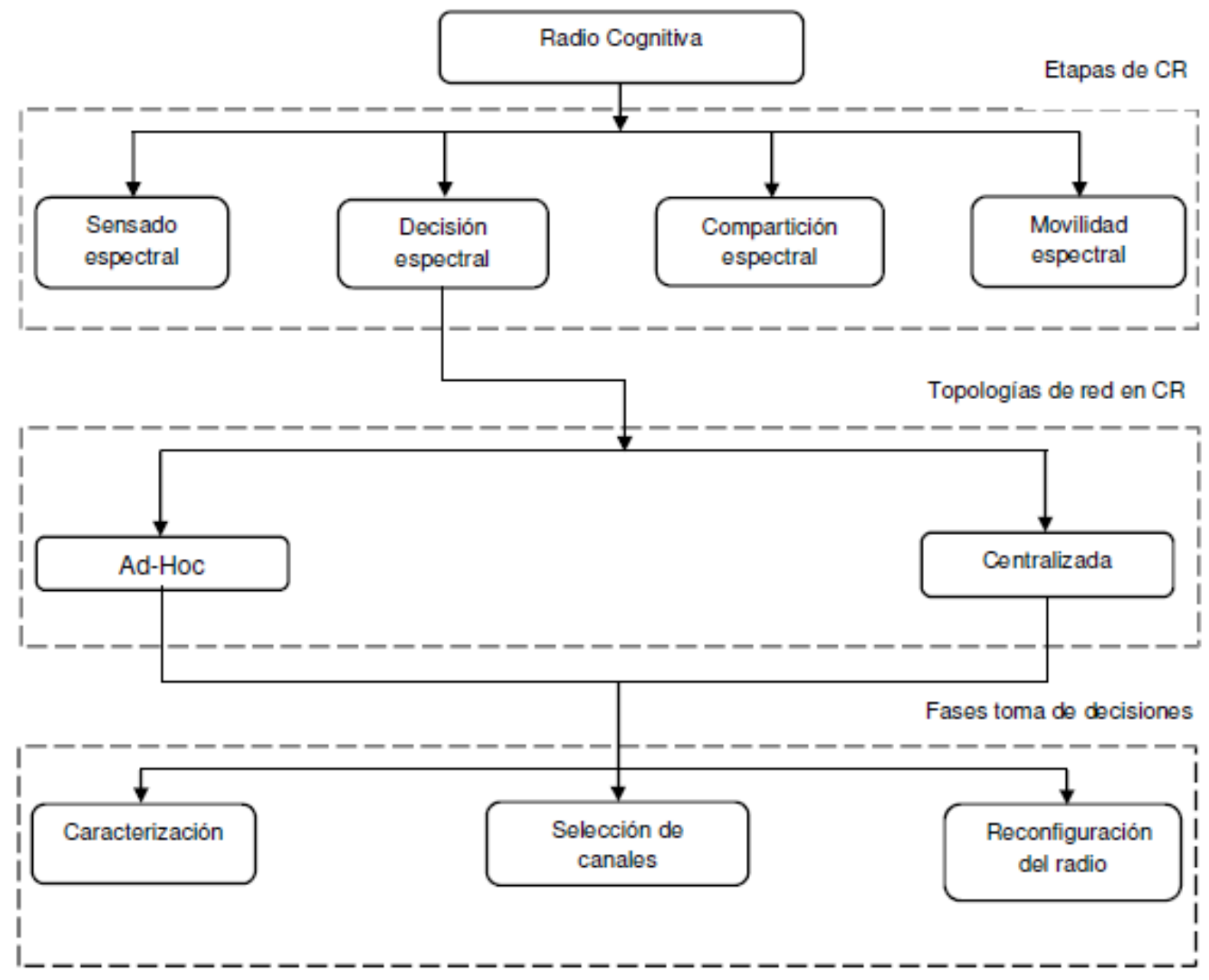

Fig. 3: Conceptualización de radio cognitiva

En una estructura Ad-Hoc, los SUs se comunican directamente entre sí, sin la necesidad de requerir de un dispositivo central que gestione; compartiendo su conocimiento local con aquellos dispositivos que se encuentren dentro del rango de alcance. A partir de su percepción de la red, cada radio puede decidirse por la selección de un canal de acuerdo a sus necesidades. Si la solicitud no es satisfecha a causa de la aparición de un PU o por degradación del canal, se tiene la posibilidad de repetir todo el proceso empezando de nuevo desde la monitorización del espectro (Kolar, et al., 2009).

En un sistema basado en infraestructura, todos los usuarios dentro de la zona de funcionamiento, gestionan sus solicitudes de decisión a través de un dispositivo central, como sucede en la especificación IEEE 802.22 (Red Inalámbrica de Área Regional-WRAN) (Wang, et al., 2011). Como la red de radio cognitiva (CRN) opera dentro de la región de cobertura de los usuarios licenciados, utiliza técnicas de DSA para acceder oportunistamente al espectro de red principal sin causar interferencia perjudicial a los licenciados al igual que en el caso Ad-Hoc. Para ello, los SUs realizan observación del espectro en las bandas especificadas, para luego enviarlas a la estación base (BS) que actúa como un centro de difusión (Kolar, et al., 2009). Tanto la BS como sus clientes asociados pueden detectar la presencia de los PUs usando técnicas de detección como el basado en energía, cicloestacionalidades, o bases de datos de geolocalización, entre otros. En algunos casos como en (Akyildiz, et al., 2011) se utilizan dos medios físicos, uno para observar el comportamiento del canal primario y el otro para actualizar constantemente la BS. Una vez analizada la información y se tenga claridad de los canales disponibles, la BS construirá la lista definitiva de los bandas junto con la calidad de servicio (QoS) disponible en cada caso, con el fin de que puedan ser aprovechados oportunistamente por los SUs. Definida la etapa previa; la caracterización, selección de canales y reconfiguración del radio son los elementos que componen la decisión espectral (figura 3). 


\section{CARACTERIZACIÓN}

El uso de bandas licenciadas por los usuarios secundarios (SUs), está condicionada a la inactividad de los canales o al no uso de los mismos por los PUs; en razón a que no hay garantía que frecuencia espectral esté disponible durante todo el periodo de trasmisión de un SU, es importante tomar en cuenta con qué frecuencia aparecen los PUs. Utilizando la habilidad de aprendizaje del radio cognitivo, la historia del uso del espectro es utilizada para predecir el perfil futuro del espectro (Masonta, et al., 2013) con la caracterización o modelaje de la actividad los PUs y SUs; de esta manera pudiéndose realizar una administración y gestión del espectro adecuado, buscando evitar la generación de colisiones con los PUs. Este planteamiento sugiere que la caracterización involucra el estudio y evaluación de tres variables importantes que son el estudio de las condiciones actuales del espectro disponible, su utilización futura por parte del PU, y el uso adecuado y sincronizado de las bandas para su aprovechamiento oportunista desde los SUs, como se muestra en la figura 4.

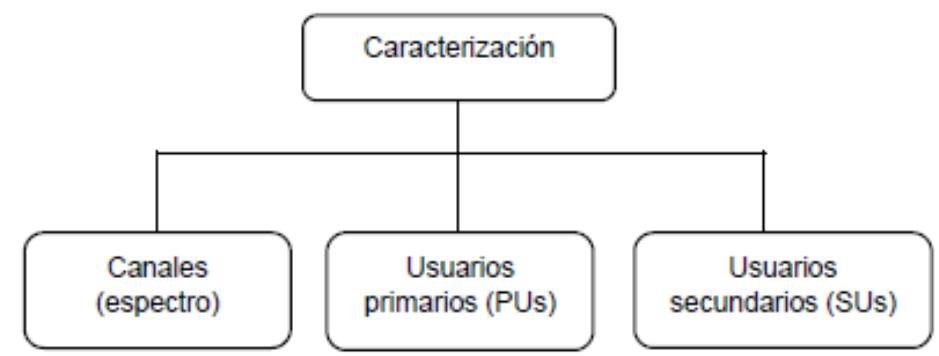

Fig. 4: Modelado de la actividad de los medios de transmisión y usuarios.

\section{CARACTERIZACIÓN DE LOS CANALES}

Bandas múltiples de espectro con diferentes características de canal pueden estar disponibles en un amplio rango de frecuencias (Won-Yeol, 2011). Determinar el medio de transmisión adecuado, involucra varios aspectos. La identificación del canal para conocer el ambiente que lo rodea, identificar las propiedades que afectan los datos en el canal considerando las aplicaciones o patrones de tráfico que soportaran las redes, sean estas determinística (redes de televisión) (IEEE 802.22, 2014) o probabilística (infraestructuras celulares) (IEEE Standard 802.22, Part 22, 2011; Almalfouh y Stber, 2011).

Estimar la interferencia de canal en ambientes CRNs con la coexistencia de PUs y SUs en los mismos rangos espectrales, evitando interferencias dañinas en los usuarios licenciados. En (Almalfouh y Stber, 2011) proponen un método de asignación de recursos de radio consciente de interferencia, estudiando la interferencia generada a un PU como consecuencia de la interferencia que aparece como resultado de la detección de espectro imperfecta. (Rabbachin, et al., 2011) propone un modelo estadístico para estudiar la interferencia ocasionada por SUs en una región limitada o finita usando protocolos de umbral individual y múltiple.

El tiempo de tenencia del canal, es la duración esperada que los SUs pueden ocupar una banda autorizada antes de ser interrumpidos; mientras mayor sea esta variable mejor será la QoS ofertado al SU. En (Yuan, et al., 2007) se introdujo el concepto de bloque espectro-tiempo para predecir el tiempo por el cual un US ocupa una porción de espectro vacante sin causar interferencia a los UPs. (Pla, et al., 2010) modela la duración del espacio espectral utilizando cadenas de Markov, para aplicar técnicas analíticas de matriz y obtener la duración de los agujeros a los que pueden acceder los USs. Las desventajas de ambas es su alto costo computacional. La caracterización del ambiente de radio frecuencia también incluye el análisis de otras diversas variables como la capacidad de canal, ancho de banda disponible, perdida de ruta, codificación que no son tratados en el artículo, pues su caracterización dentro de la propuesta se asume conocida, ya que no es tema de interés en razón a la cantidad de publicaciones y soluciones existentes (Xu, et al, 2008; Matinmikko, et al., 2008; Azarfar, et al., 2012; IEEE Standard, 2011), entre otras.

\section{CARACTERIZACIÓN DEL PU}

La estimación futura de la ocupación de los canales por parte de los PUs da un indicio a los SUs de los momentos en los que se podrá hacer uso del espectro para transmitir; métrica considerada como sensible y que va a depender de que tan exacto es el modelo de predicción a partir del histórico de utilización del mismo.

En la caracterización de los primarios, (Vishram, et al., 2012) concluye que un número importante de los planteamientos existentes tienen un costo computacional muy alto, haciendo prácticamente inviable su 
implementación en aquellos nodos que basan su vida útil en el uso de baterías (dentro de zonas rurales); planteamiento que sugiere que a pesar de ser un tema abordado por varios investigadores, sigue presenta varios desafíos de desarrollo en el sentido que es necesario la propuesta de metodologías que reduzcan el costo computacional al estimar futuras predicciones a partir de los datos existentes. Las técnicas más representativas que estudian la dinámica de los PUs se resumen en la figura 5.

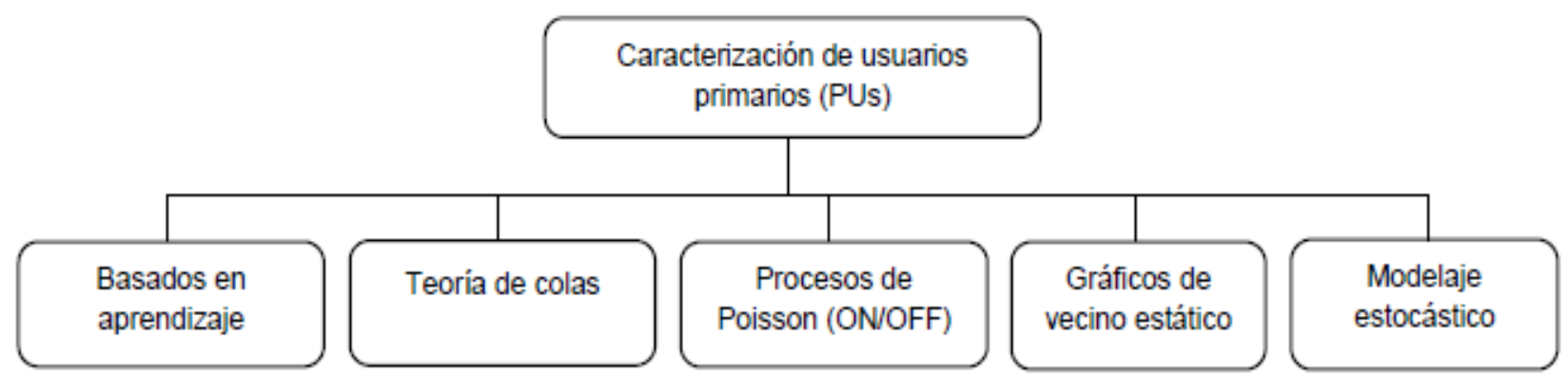

Fig. 5: Principales paradigmas usados en la actividad de los PUs.

En (Husain, et al., 2013) se formula una estrategia para la selección inteligente de canales en CR multi-salto (SURF); su principio de funcionamiento se beneficia de las cadenas de Markov en tiempo continuo para clasificar el espectro disponible bajo la suposición de una baja actividad en los PUs y alto número de nodos cognitivos, provocando que cada SU dinámicamente migre al mejor canal. Se incluye un ciclo en el algoritmo para aprender de aquellas estimaciones donde la selección de la banda fue equivocada, retroalimentando ese aprendizaje en futuras predicciones. Se concluye que si el nivel de actividad del PU es alto, la solución planteada no es adecuada, resultado lógico ya que hay deficiencia de huecos espectrales libres; cuando es intermitente, la estrategia de selección funciona bien mejorando el rendimiento del sistema a través del control de colisiones con los PUs.

Un estudio analítico para una red hibrida basada en el estándar IEEE 802.11 es abordado en (Khabazian, et al., 2012), Para preservar la prioridad de los PUs, se supuso que los SUs disputan su aprovechamiento cuando se encuentre libre de cualquier actividad del primario durante un periodo de tiempo.

Usan la teoría de colas y modelan la variabilidad de los nodos licenciados. En este caso cada PU se estructura como un sistema de encolamiento discreto M/G/1 con tasa de arribo de $\lambda$ paquetes/seg y de servicio de $1 / E[D]$, con $E[D]$ como el retardo de acceso al medio de los PUs en presencia de los SUs, y su valor muestra la media del intervalo de tiempo entre el instante de arribo de un paquete a la cola principal de un PU y el instante de tiempo en el que ese PU accede al canal para realizar la transmisión. El valor de esta variable asociada con el retardo de encolamiento incide directamente en la obtención del retardo promedio de los paquetes $E[D t]$ mostrado en la ecuación 1.

$$
E\left[D_{t}\right]=E[D]+\frac{\lambda E\left[D^{2}\right]}{2(1-\lambda E[D])}
$$

donde, $E\left[D^{2}\right]$ denota el segundo momento de tiempo que tarda un PU en acceder al medio, y $\lambda E[D]$ es la carga existente en la cola. El análisis de las simulaciones aduce que el desempeño de la red primaria con una tasa de llegada de paquetes dado, puede verse afectado dependiendo del tamaño de la carga útil del paquete y de la cantidad de nodos SUs vecinos.

El enfoque estadístico basado en series temporales binarias en (Yarkan y Arslan, 2007), da a conocer el comportamiento determinista y no determinista de uso del canal para predecir la ocupación futura del PU. La complejidad del análisis y la cantidad de memoria de almacenamiento necesario (de los datos) lo reducen suponiendo una secuencia de estados binarios, simplificando así también la ocupación del espectro ("1" es vacío, "0" es utilizado). De las pruebas realizadas el factor de predicción de rango corto es bastante satisfactorio para las dos primeras pruebas realizadas, sin embargo en la tercer muestra el éxito de la predicción se degrada fuertemente, porque el modelo no se actualiza, además de que el comportamiento de los datos es no determinístico; problema que teóricamente podría resolverse incrementando su orden, a expensas de un aumento exponencial de los parámetros para generar la predicción. Desde la perspectiva determinista, la estimación es bastante robusta para las cuatro primeras ranuras de tiempo según las pruebas realizadas para tres bandas distintas en una red GSM durante una captura con una duración de 17 $\mathrm{ms}$, de la que se obtuvieron 30 observaciones por canal una vez aplicado el modelo. 
(Vishram, et al., 2012) plantea un sistema de decisión espectral decisiones en el espectro basado en la calidad de servicio para redes CR, que garantiza el tratamiento adecuado de los paquetes mediante la ubicación de una banda capaz de satisfacer los requerimientos del SU, donde estos pueden generar tráfico con múltiples prioridades, dividiendo los flujos a procesar en 4 diferentes tipos, con 8 bandas espectrales disponibles teniendo en cuenta además cómo variables del sistema la disponibilidad del canal, la fluctuación del usuario primario; con el supuesto que el ancho de banda es el mismo en cada caso (condición que podría ser una ventaja si se configura el sistema para que opere así). Se modela la disponibilidad del canal como una fuente ON/OFF dependiendo de la presencia o ausencia del PU (que posee un patrón de comportamiento conocido) con cadena Markoviana de dos estados. Los parámetros $\alpha$ y $\beta$, representan la probabilidad de transición del PU en el canal, dado un estado ON (presencia) a uno OFF (ausencia) y viceversa. La probabilidad de disponibilidad de un agujero está dada por:

$\prod_{i}=\frac{\alpha_{i}}{\alpha_{i}+\beta_{i}} \forall i \in C^{\prime}$

dado que: $C^{\prime}=\{1 \ldots C\}$. Del análisis de resultados se encuentra que cuando el número de canales aumenta, la aparición de falsa alarma disminuye haciendo que el sistema funcione más adecuadamente.

El predictor basado en el gráfico del vecino estático (SNG) (Xing, et al., 2013), se diseña para predecir las futuras ubicaciones de los PUs de acuerdo con la información previa recogida de la topología de movilidad de los mismos usuarios licenciados. Inicialmente, se construye un gráfico orientado a representar el historial de la movilidad de los PUs. Para ello cuando un usuario secundario observa el paso de un PU de la ubicación $i$ a la $j$, se añade una arista dirigida $(i, j)$ a la gráfica y se establece el peso de la arista para $\omega_{i j}=1$ si la arista $(i, j)$ no está en el gráfico, o se añade 1 al peso de la arista, $\omega_{i j}=\omega_{i j}+1$ si la arista $(i, j)$ está en el gráfico. Una vez obtenida la gráfica, se realiza un procedimiento de normalización en los pesos de las aristas de manera que $\forall i, \sum_{j} \omega_{i j}=1$. Luego la movilidad de los PU se predice de la siguiente manera: $\mathrm{Si}$ la ubicación actual del UP es $i$, y el usuario cognitivo encuentra el lugar $i$ en el gráfico, el devuelve una lista $\left(j, \omega_{i j}\right)$ para todas las aristas $(i, j)$ y, a continuación predice la futura ubicación del PU como $j=\arg \max \omega_{i j}$ (Butun, et al., 2012). Un aspecto interesante predecir el PU basado en SNG es que se puede obtener información valiosa adicional de la estructura de la red.

En (Ghosh, et al., 2010), se propone un modelo estadístico de variación de tiempo para ocupación del espectro utilizando medidas de frecuencia reales. Usando características estadísticas extraídas de medidas de RF reales, se emplean parámetros de primer y segundo orden en un estándar estadístico de ocupación de espectro basado en una combinación de funciones de probabilidad diferente de densidad (Masonta, et al., 2013).

Aunque la mayor parte de las investigaciones sobre radio cognitiva (CR) se centran en bandas de frecuencia por encima del límite superior de altas frecuencias (HF) (Gutiérrez, et al., 2013), los principios de CR también se pueden aplicar a comunicaciones en la banda de las altas frecuencias (HF) para hacer un uso más adecuado del espectro, partiendo de las restricciones regulatorias y de propagación. En este trabajo se considera a los usuarios heredados de otras frecuencias como los PUs que transmiten sin recurrir a ningún procedimiento inteligente y se usa la arquitectura HFDVL (transporte de voz y datos en HF usando anchos de banda de $3 \mathrm{KHz}$ ) como SUs. El objetivo de estudio es mejorar la eficiencia en el uso del espectro detectando la presencia futura de los PUs en los canales (para evitar colisiones), mientras se transmite la información de los SUs en diferentes canales usando el transceptor HFDVL. Para ello un algoritmo dinámico que se encarga de monitorear la actividad de los PUs (Wang, et al., 2011) es desarrollado estimando las predicciones futuras a corto plazo del tiempo de permanencia utilizando el modelo oculto de Markov (HMM). El sistema es entrenado para valores reales obtenidos en la banda de radio aficionado en la frecuencia de los $14 \mathrm{MHz}$ en tres situaciones diferentes: canales disponibles, parcialmente disponibles y no disponibles. La validación de resultados se basó en predecir la actividad en un canal durante el siguiente minuto, alcanzando un error de predicción promedio igual a $10.3 \%$ cuando el conocimiento previo de la actividad en el mismo es de un minuto de duración, pudiendo disminuir su valor a un $5.8 \%$ cuando el tiempo previo de análisis es de 8 minutos.

En CRNs, un modelo estático como el mostrado en (Song y Zhang, 2010) (donde usan solo un ciclo para el sensado de la actividad e inactividad de los PUs) no logra capturar la dinámica del comportamiento de los PUs en el dominio temporal. En (Ning, 2013) se los Autores incrementan el número de mediciones (a dos ciclos) para estimar con precisión la actividad del PU y así posteriormente poder mejorar el desempeño de los algoritmos de detección de bandas libres. El método propuesto conmuta entre dos opciones; el primero llamado de sensado fino (que se utiliza cuando el SU ingresa por primera vez a la red primaria, y por tanto ignora el funcionamiento del PU), y el segundo de sensado normal; que apoyados en 
un estimador de máxima verosimilitud, aprenden a conocer los periodos de actividad e inactividad del PU (en la detección fina). Estos patrones de ocupación son usados en la detección normal, donde el valor del error cuadrático medio (MSE) de los periodos ON-OFF es continuamente monitoreado para asegurar una estimación suficiente precisa. Cuando su actividad cambia significativamente (es decir el valor del MSE es superior a un umbral) obliga a ejecutarse de nuevo el sensado fino. Los resultados en la simulación muestran que el método sigue la dinámica de la actividad del PU aun en altos niveles de fluctuación ara el PU.

La literatura científica fundamenta la representación de la actividad de los usuarios primarios con metodologías que tienen un costo computacional importante como (Khabazian, et al, 2012), (Yarkan y Arslan, 20107), (Xing, et al., 2011)), siendo inviables en aplicaciones de campo abierto cuando la conservación de la energía es importante; los procesos de Poisson también con las desventajas planteadas en (Canberk, et al, 2011); y los basados en esquemas estadísticos que son modelos muy idealizados volviendo inviable su aplicación real. Una alternativa, que pudiera resolver estas falencias incrementando la eficiencia, son los modelos basados en aprendizaje autónomo que se retroalimentan de sus propios errores para mejorar las prestaciones en el futuro como en (Husain, et al., 2013).

\section{CARACTERIZACIÓN DEL SU}

Uno de los aspectos críticos y que cuenta con menos desarrollo se relaciona con los secundarios, la literatura científica reduce su estudio a un problema de control de acceso al medio, relegando en la mayoría de los casos a un segundo plano la importancia del estudio de la frecuencia de aparición de los SUs en la banda licenciada, cuando la cantidad de SUs que arriban a la red licenciada tiene un comportamiento exponencial creciente (caso que es muy probable encontrarse en la vida real). A partir de la literatura existente, dentro de las metodologías aplicadas a su caracterización (figura 6) y representación se encuentra el estudio de la conducta de los SUs abordado en (Csurgai y Bito, 2011) denotando su actividad con cadenas Markovianas de dos estados (OFF se presume inactividad, y ON uso del canal), donde el flujo de datos que genera el tráfico en los nodos cognitivos es del tipo Internet (chat, email, transferencia de archivos), con la matriz de transición de la ecuación 3).

$$
p=\left(\begin{array}{ll}
p_{11} & p_{21} \\
p_{21} & p_{22}
\end{array}\right)
$$

donde, $p_{i, j}$ es la probabilidad que estando en el estado $i$ se vaya al estado $j$, y su cálculo se obtuvo de la utilización de una representación estadística de tráfico publicado en (Harris, 2013), llegándose a encontrar los siguientes valores teniendo en cuenta un periodo de muestreo de 13 horas/semana:

$$
p_{11}=0.999997 ; p_{12}=0.000003 ; p_{21}=0.99980 ; p_{22}=0.00020
$$

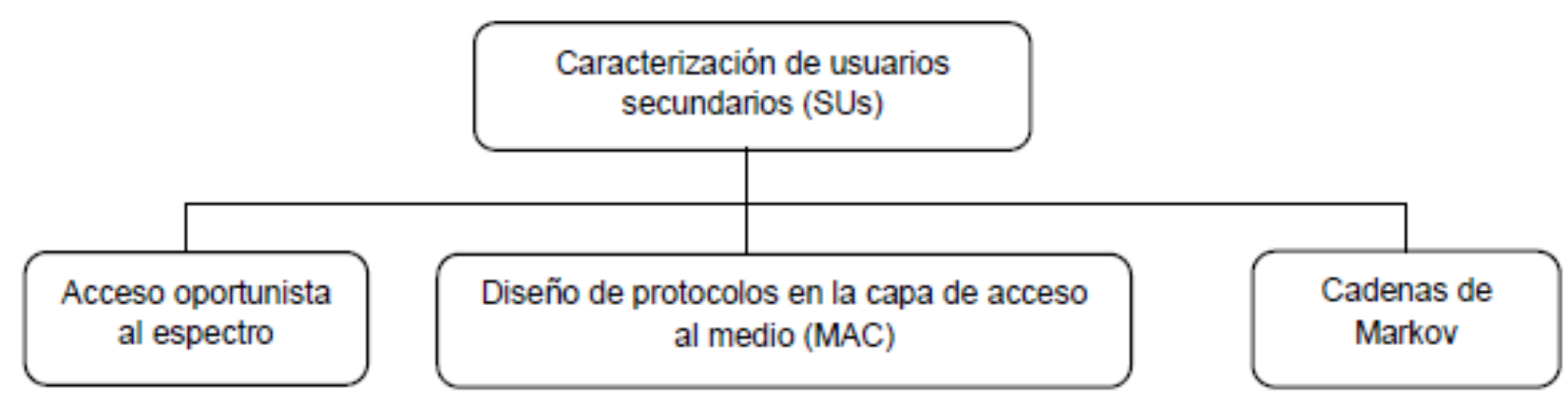

Fig. 6: Principales arquetipos para monitoreo de SUs.

Una representación gráfica de las probabilidades de cambio de estado se muestra en la figura 7; el modelo presume que los parámetros son variables y siguen una distribución normal muy cercana al comportamiento real de los usuarios, donde la media $\mu=p_{i, j}$ es la probabilidad de transición, y la varianza $\sigma^{2}$ puede ser calculada, tomando la desviación estándar del uso semanal de los datos. 


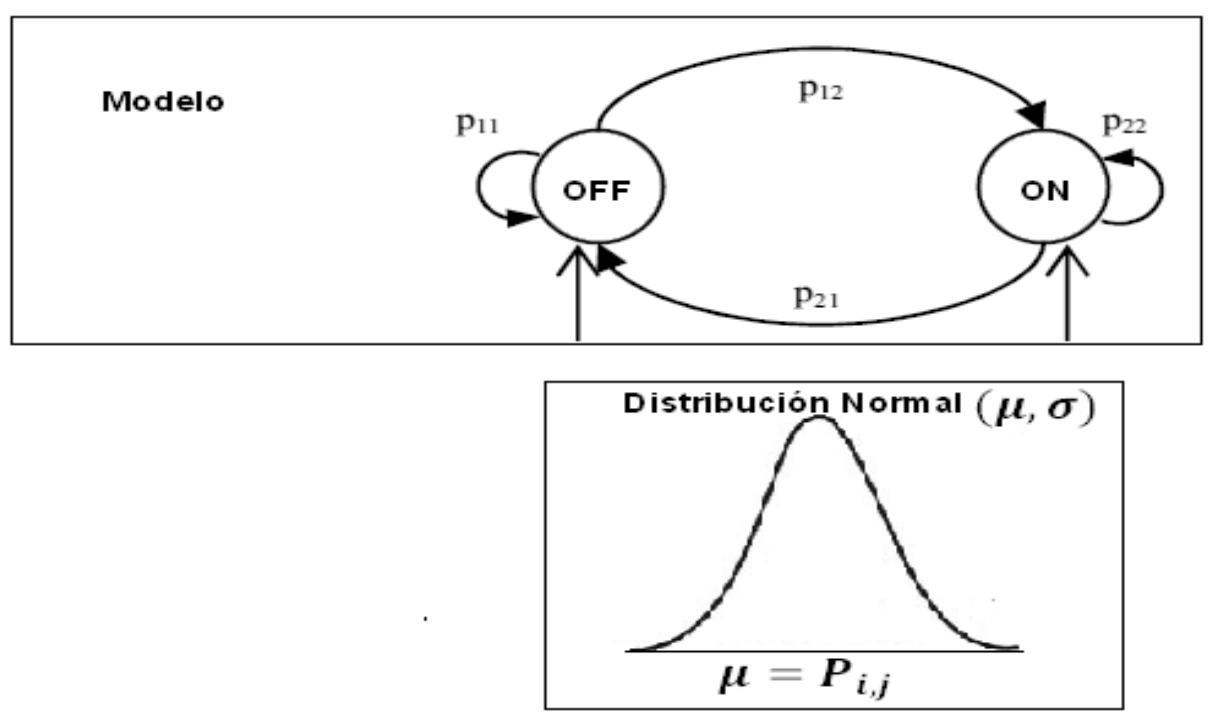

Fig. 7: Modelo del SU (Csurgai y Bito, 2011).

En (Derakhshani y Le-Ngoc, 2013) Consideran una estrategia de transmisión de salto adaptativo para nodos cognitivos como estrategia temporal de acceso a franjas de espectro libre en la banda licenciada teniendo como punto de partida un acceso aleatorio de los PUs. Los SUs tienen la capacidad de trasladarse dinámicamente sobre espacios espectrales inactivos, con un factor de adaptabilidad que disminuye el riesgo de pérdida masiva de información en caso que el PU desee usar la banda licenciada. Para ello, el problema de optimización que se plantea es crear un algoritmo de acceso al espectro oportunista (OSA) que beneficie el SU, supeditado a oportunidades espectrales existentes con la descomposición dual de Lagrange; buscando que en lugar de detectar y seleccionar frecuencias en desuso (como en otros estudios), se pueda acceder a múltiples canales probablemente con tiempos de permanencia diferentes, en el que cada SU autónomamente adapte sus factores de movimiento a valores óptimos.

De los artículos de corriente principal solo (Derakhshani y Le-Ngoc, 2013) presenta una propuesta que incluye un análisis cualitativo y cuantitativo, para mejorar el aprovechamiento de las bandas libres a partir de la utilización de metodologías oportunistas de acceso al espectro. Tal apreciación sustenta la escasa existencia de modelos inteligentes basados en nuevas herramientas matemáticas como lenguaje de máquina (ML), y máquinas de soporte vectorial (SVM), que optimicen el aprovechamiento del espectro licenciado disminuyendo la probabilidad de interferencia con otros usuarios de la red.

\section{SELECCIÓN DE CANALES}

Una vez caracterizados los usuarios, el siguiente paso es elegir el mejor espectro disponible (dependiendo de la topología de red usada; se utilizara una red centralizada (figura 3)) para ser asignados de acuerdo con los requisitos solicitados por los SUs (Masonta, et al., 2013) usando una estrategia reactiva o proactiva. La figura 8 resume las técnicas de selección reactiva que se han aplicado a la conceptualización de la propuesta.. Hasta el momento la literatura científica no han sido propuestos métodos que apliquen el concepto proactivo. Este traería como beneficio una disminución en el tiempo de asignación de bandas, disminuyendo el tamaño en la cola de espera de usuarios cognitivos en topologías fisicas centralizadas.

(Suraweera, et al., 2010) Compara las selecciones de canal de mejor ajuste (BFC) y de canal con el tiempo de inactividad más amplio (LITC) desde las perspectivas del rendimiento de la red (en una infraestructura centralizada), fragmentación espectral, probabilidad de éxito en la transmisión, utilización oportuna del espectro para validar las mejoras hechas a la técnica BFC. BFC, tiene en cuenta la actividad de tráfico en el canal del SU y PU, implícitamente cuenta con un mecanismo eficiente de reparto del espectro que mitiga los efectos de la fragmentación espectral, factor que degrada significativamente el uso del espacio radioeléctrico en CR. LITC fundamenta su escogencia en aquellas bandas con tiempos de inactividad más amplios, independientemente de los requisitos de transmisión, maximizando su beneficio propio independientemente si hay derroche de recursos que pudieran ser utilizados por otros. Una serie de simulaciones muestran la superioridad de BFC en términos de la probabilidad de éxito en la transmisión, utilización oportuna del espectro. 
Lindberg (2012), propone la lógica difusa para la ubicación y asignación del espectro más adecuado, evitando aquellos canales que los usuarios primarios utilizan con frecuencia y que puedan degradar el rendimiento del sistema. El sistema jerárquico propuesto (figura 9) consiste en dos controladores difusos (FLS).

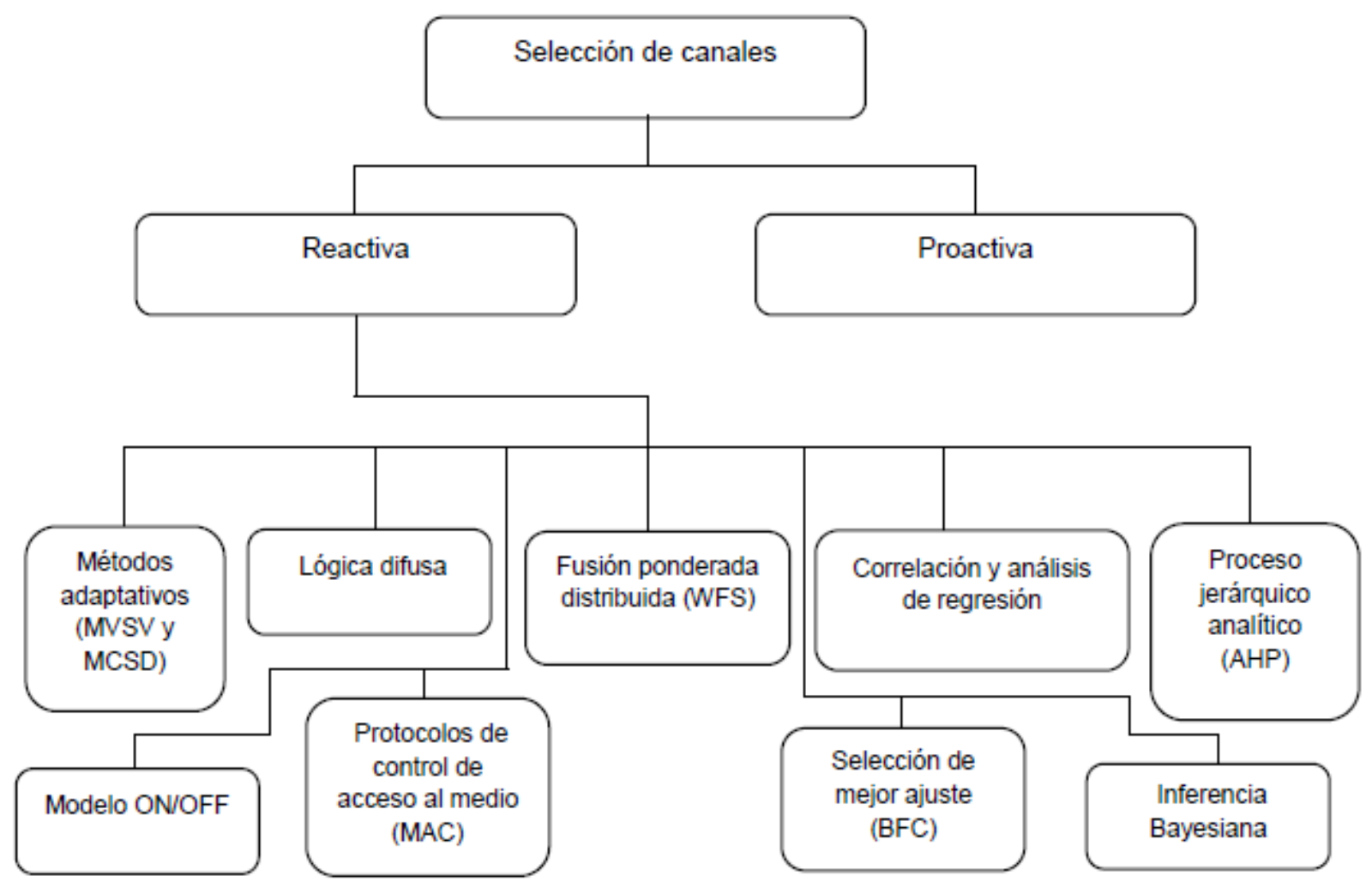

Fig. 8: Metodologías de selección de canales.

EI FLS1 recibe tres entradas: la eficiencia espectral, distancia y movilidad, y entrega la probabilidad de utilización del canal; el FLS2 manipula tres entradas: la probabilidad de la utilización del canal, la tasa de habilitación del canal, y la tasa de no disponibilidad del canal, y define la posibilidad de asignar un canal para el acceso del usuario secundario al espectro. La asignación del espectro, se lleva a cabo considerando la calidad de servicio en términos la rata de error de bit, mediante el motor de inferencia comparando la medida de la relación señal-interferencia-ruido para diferentes periodos en los canales disponibles.

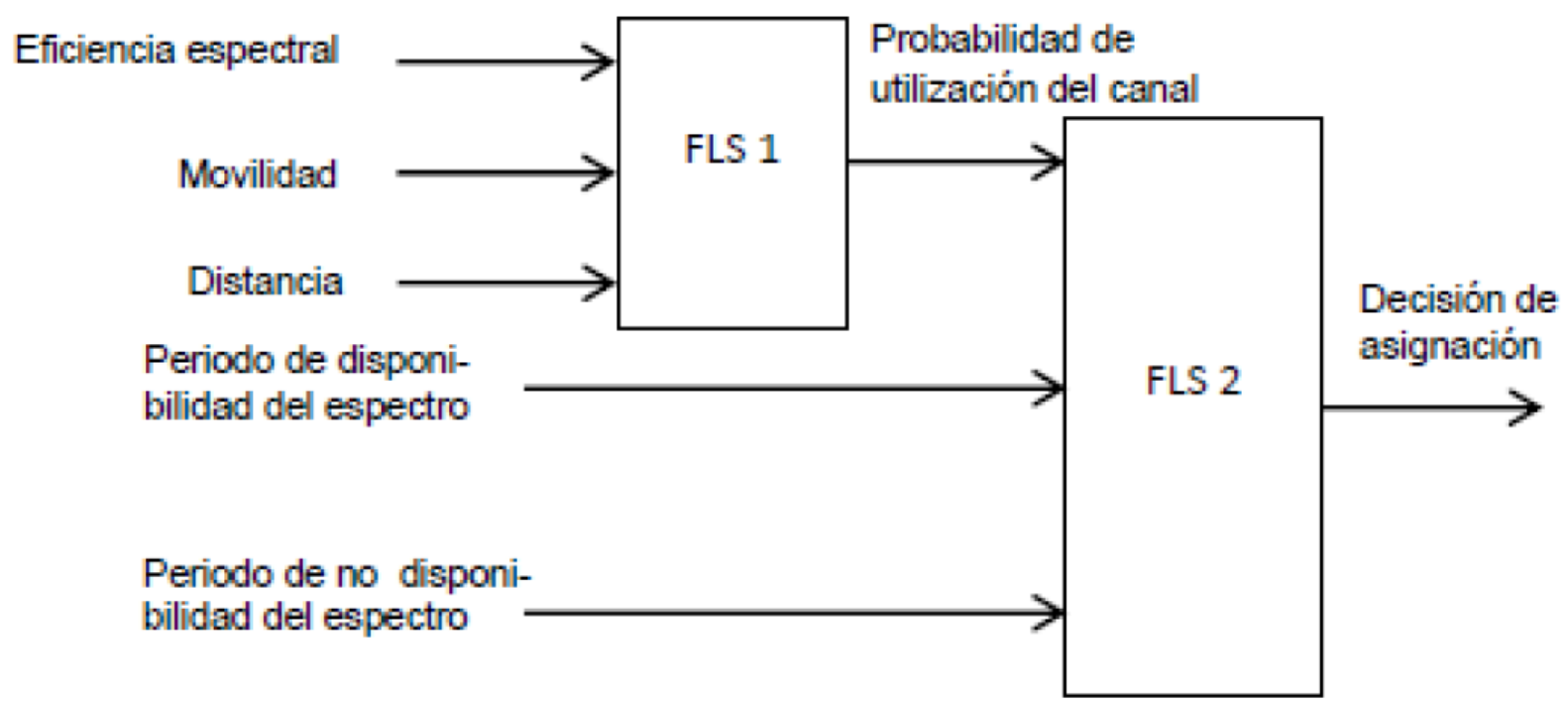

Fig. 9: Controladores difusos jerárquicos (Lindberg, 2012). 
El aporte de (Xiaoshuang, et al., 2013) se plantea en términos del paradigma probabilístico de inferencia Bayesiana (BIF), que busca predecir la calidad del canal en CRNs en función de la probabilidad de falsa alarma y el tiempo de duración en estado inactivo de la banda en una networking centralizada. Procesada la información es clasificada en orden descendente de acuerdo a las cuantificaciones obtenidas en (Xing, et al., 2013) para posteriormente otorgar las frecuencias. La idea es innovadora porque en ningún caso se ha considerado la importancia de la duración del periodo de inactividad; por ejemplo en (Nie y Comaniciu, 2005), (Niyato y Hossain, 2008) se modela la utilidad de los usuarios secundarios como función de la calidad del canal pero no se discute como evaluarla en términos de esta variable. Los Autores estiman el tiempo de inactividad, definiendo la probabilidad de detección $P d$ y falsa alarma $P f$ como en las ecuaciones 4 y 5 .

$$
\begin{aligned}
& P_{d}=e^{-\frac{x}{2}} \sum_{n=0}^{m-2} \frac{1}{n !}\left(\frac{x}{2}\right)^{n}+\left(\frac{1+\xi}{\xi}\right)^{m-1} \cdot\left[e^{-\frac{x}{2(1+\xi)}}-e^{-\frac{x}{2}} \sum_{n=0}^{m-2} \frac{1}{n !}\left(\frac{x(1+\xi}{2(1+\xi)}\right)^{n}\right] \\
& P_{f}=\frac{\Gamma\left(m, \frac{x}{2}\right)}{\Gamma(\mu)}
\end{aligned}
$$

donde $x$ es el umbral del detector de energía, $m$ el producto entre el ancho de banda y el tiempo, $\xi$ la relación señal a ruido promedio del $\mathrm{SU}$ en el canal primario, $\Gamma(.,$.$) la función incompleta gamma y \Gamma($.$) la$ función gamma. Es preciso tener en cuenta que $\xi=P_{P U} h_{P S} / \zeta^{2}$, con $P_{P U}$ indicando la potencia de transmisión del primario, 5 como la varianza del ruido Gaussiano y $h_{P S}=k / d_{P S}^{\mu}$ como la pérdida de trayectoria entre el SU y PU (aquí $k$ es la constante de pérdida de trayectoria, $\mu$ es el exponente de pérdida de trayectoria, $d_{P S}$ la distancia entre el PU y SU). La calidad del canal $\left(M_{Q}\right)$ se encuentra combinando las métricas de precisión de la detección de espectro $M_{A}$ y la duración estimada de inactividad en el canal $M_{E}$ (ecuación 6).

$$
M_{Q}=\left(1+\log _{e} M_{A}\right) M_{E}
$$

e $>1$ es un parámetro de la preferencia del SU cuando se estima la calidad del canal licenciado.

En (Won-Yeol y Akyldiz, 2011) se presenta un marco de decisión para determinar un conjunto de bandas, teniendo en cuenta los canales dinámicos en las redes RC, así como los requisitos de aplicación. Para este fin, primero, se propone un nuevo modelo de la capacidad del espectro que considera características únicas en redes CR. Basado en este modelo de capacidad, el MVSD está desarrollado para aplicaciones en tiempo real, que determina las bandas de espectro para reducir la capacidad de variación. Para las aplicaciones de mejor esfuerzo, se propone un MCSD donde las bandas del espectro deciden maximizar la capacidad total. Por otra parte, un sistema de gestión dinámica de recursos se introdujo para permitir que la red coordine la decisión de espectro adaptativo que depende de los recursos del espectro que continuamente varia en el tiempo. Los resultados de simulación muestran que el sistema de decisión de espectro propuesto prevé la utilización eficiente de ancho de banda al tiempo que garantiza la calidad del servicio.

Rodríguez, et al. (2011) afirman que la detección y decisión son de las tareas más importantes que la radio cognitiva debe realizar con el fin de seleccionar la mejor banda posible. El proceso jerárquico analítico (AHP) se considera en aras de encontrar el canal adecuado dentro de un número finito de opciones, incluyendo varios atributos en orden de importancia y dependientes del nivel inmediatamente anterior (figura 10). Una de las formas de validar la propuesta fue estudiando la variación de la latencia en función de las oportunidades espectrales. Aunque en el trabajo presentado no específica la topología, se intuye que es centralizada por la arquitectura planteada.

Algunos de los problemas de investigación abiertos en la selección y asignación de espectro son: La utilización y aplicación de paradigmas inteligentes y de aprendizaje autónomo aplicados a diseñar sistemas cognitivos usando una estrategia proactiva (este tipo de modelos son inexistentes de acuerdo con la teoría revisada) buscando minimizar los tiempos de asignación; la selección cooperativa buscando maneras eficientes de combinar la información de los nodos cognitivos cooperantes mientras se aborda la sobrecarga de trasmisión y/o cooperación (Masonta, et al., 2013).

\section{RECONFIGURACIÓN DEL RADIO}

El hardware de las redes inalámbricas tradicionales está configurado estáticamente para funcionar sobre frecuencias previamente establecidas. Aunque estas tecnologías pueden emplear aplicaciones remotas 
para ajustar algunos parámetros como ancho de banda, encriptamiento, potencia de transmisión y recepción, su arquitectura física y lógica limita la flexibilidad para adaptarse a un ambiente externo. Con la radio cognitiva esta habilidad de adaptación autónoma es la propiedad más desarrollada (pues es su razón de existir), adaptando sus parámetros de funcionamiento supeditado a las exigencias del SU y en concordancia con el ambiente local existente. Por no ser tema incluido en la propuesta no es abordado.

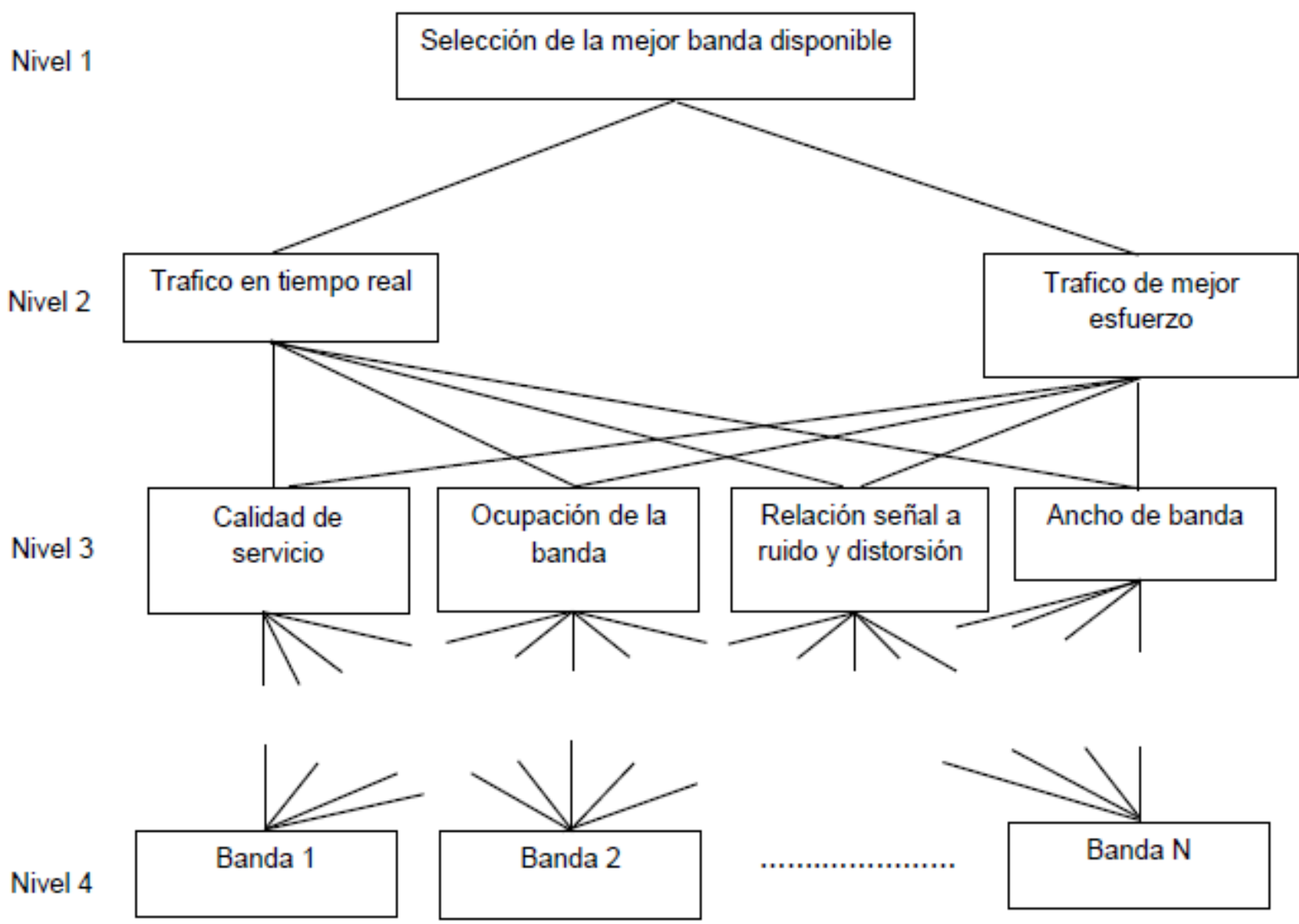

Fig. 10: Niveles jerárquicos definidos en AHP (Rodríguez, et al., 2011).

\section{CONTEXTUALIZACION DEL PROBLEMA}

Partiendo del marco conceptual generado y mostrado anteriormente desde la situación actual de la fase de toma de decisiones espectral en CR, se debe destacar que aunque la fase inicial de sensado de espectro en radio cognitiva está plenamente desarrollada y soportada en múltiples modelos como bases de datos de geolocalización; la etapa de toma de decisiones aún se encuentra en un ciclo temprano de maduración, esto debido en mayor parte a que la literatura disponible se centra en el modelaje de la actividad del PU e ignora el modelamiento del SU (Masonta, et al., 2013), además de que los modelos propuestos para pronosticar el estado futuro del comportamiento de los PUs tienen un costo computacional muy alto que hace imposible su implementación real sobre todo en aquellas situaciones donde la carga de la batería del nodo es un factor sensible (zonas rurales).

Estas condiciones, además de que se hace necesario incluir nuevas técnicas de modelaje como las basadas en inteligencia artificial (IA) en la selección anticipada y adecuada de canales a ser usados por SUs para el transporte de datos, buscando reducir los tiempos de asignación de bandas espectrales, son algunos de los problemas que enfrenta la CR y que han impedido que actualmente existan equipos comerciales confiables que hagan realidad la aplicación del paradigma de la radio cognitiva desde el punto de vista real. En este sentido el problema a resolver se centra en los módulos de color oscuro de la figura 11 , donde lo que se pretende es proponer a la comunidad científica un sistema de toma de decisiones que tiene como objetivo final lograr un sistema que logre reducir los tiempos de selección de canales a usuarios no licenciados utilizando técnicas inteligentes de autoaprendizaje basadas en IA, a partir de la determinación de la caracterización del PU y el SU. 


\section{PROPUESTA DE ASIGNACIÓN DE BANDAS}

En la figura 12 se presenta el modelo conceptual de la propuesta de asignación de bandas espectrales en redes inalámbricas de radio cognitiva. El modelo está centrado en:

Caracterizar usuarios secundarios. Diseñar de un algoritmo (a partir del uso histórico del canal), para la asignación equitativa de la banda espectral en una red cognitiva.

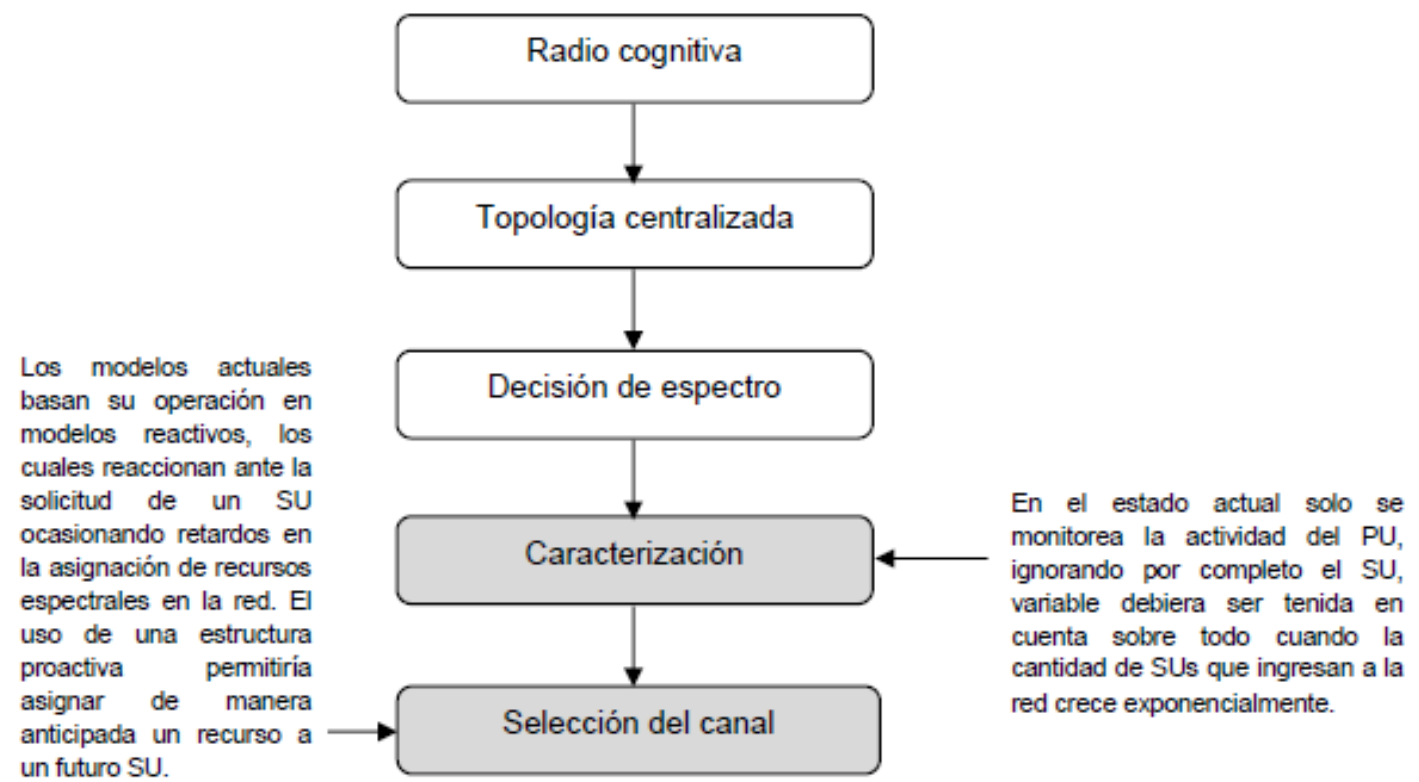

Fig. 11: Ubicación del problema dentro del contexto de la CR.

Selección de bandas usando una estrategia proactiva. Construcción de un modelo predictor en el que el algoritmo sea capaz anticipadamente de elegir la banda (dependiendo del tráfico del SU) a ser otorgada en un futuro.

Inicialmente se asume que la topología de trabajo será centralizada, y que existen oportunidades espectrales disponibles para soportar las transmisiones (es decir existe un sensado de espectro), el esquema de ocupación del secundario sobre el que se tiene estimado trabajar con respecto al primario es de tipo overlay o de superposición espectral.

Los aportes de la propuesta están diferenciados en color oscuro (figura 12). Específicamente la propuesta parte de la existencia de un número finito de usuarios SUs y PU (la cantidad de nodos SUs se irá incrementando para evaluar el modelo). Los datos para los SUs se obtendrán de fuentes de tráfico disponibles en la web, otra alternativa es generar con algún tipo de distribución. La fuente de datos del PU será a ráfagas con la propiedad de que el ancho de banda de transmisión y potencia de transmisión serán siempre iguales. Para la caracterización del PU, se modelara alguna de las soluciones existentes disponibles en la literatura científica y su identificación se realizará sobre el modelo de tráfico del mismo, lo que permitirá estimar la ocupación de los canales. Como se deben diferenciar los SUs para llevar un registro del histórico de uso del canal de manera individual, se usará una base de datos y diferenciar el tráfico de cada SU con etiquetado, otra alternativa es realizar ese control desde la capa MAC con una estándar que incluya un campo de identificación.

En la selección del canal, se desarrollará un modelo con una estrategia proactiva para estimar la probabilidad de aparición de un $\mathrm{SU}_{\mathrm{N}}$ con una aplicación específica (a partir del historial existente en la base de datos), adelantándose a la selección de la banda que será asignada en un futuro. La tarea principal de este algoritmo, es reducir el tiempo que tarda en ser escogido el canal adecuado, teniendo en cuenta la no interferencia del PU para la variable potencia de transmisión (parámetro que a nivel práctico se garantiza estableciendo criterios de máxima potencia de transmisión en la estación base). El algoritmo clasificador, será el responsable de distribuir equitativamente las banda espectrales disponibles, dando la oportunidad de ser usada por diferentes SUs de manera ecuánime. 
La prueba de funcionamiento del sistema, se estima realizarse mediante el incremento gradual en la cantidad de SUs, para ir corroborando el número de predicciones exitosos; determinando así hasta qué punto es óptima la propuesta y con qué porcentaje de acierto.

Desde el punto de vista de la base de datos, el esquema propuesto para la persistencia de los datos, está basado inicialmente en un modelo NoSQL (es decir no relacional) (Lau, et al., 2011) integrado con Map Reduce (framewok escrito en java para consolidación de información). NoSQL es libre de esquemas, lo que permite su manejo de una manera dinámica, facilitando la escalabilidad horizontal y la adición de nodos cognitivos y primarios a medida que estos arriben a la CRN sin afectar el sistema. Si se utilizara un modelo relacional convencional sería muy difícil que el sistema pudiera ser escalable debido a múltiples factores como el costo de diseño, implementación e interrelación entre la caracterización y la decisión espectral final. Particularmente un tipo NoSQL a utilizar es Object Database, que integra la base de datos con lenguajes de programación orientado a objetos como Java, permitiendo el encapsulamiento, la persistencia de las operaciones, integridad de los datos o concurrencia, gestión de almacenamiento secundario y recuperación, e interacción entre los bloques que hacen parte de la solución a la problemática dentro de la toma de decisiones.

Para la validación del sistema, se pretende comparar el algoritmo final con otro predictor existente en la literatura científica (sin tener que llegar a implementarlo), contrastando los resultados existentes publicados con los encontrados en la investigación como (Timmers, et al., 2011), (Xing, et al., 2013), (Motani y Liang, 2013). Otra alternativa para validar los resultados incluye realizar una transmisión desde un SU emisor a un SU receptor y confrontar la cantidad de datos que están arribando al destino, frente a los que se están enviando desde la fuente, o establecer la cantidad de predicciones exitosas generadas a medida que se va incrementando el número de SUs en la CRN.

Se tiene previsto que los resultados finales serán los algoritmos de caracterización, predicción y clasificación de acuerdo con los parámetros establecidos. Finalmente los paradigmas de programación viables de utilizar por sus propiedades de autoaprendizaje y bajo procesamiento computacional son FAHP, IA y ML.

FAHP (Proceso Jerárquico Analítico extendido con lógica difusa). Es un tipo de paradigma de programación con capacidad de involucrar un número importante de atributos y variables de tipo cualitativo y cuantitativo jerárquico o por niveles facilitando el control de múltiples métricas. Ha sido utilizado en la toma de decisiones en las áreas de ingeniería de software (en la verificación del cumplimiento de requerimientos de diseño), e industrial (en la selección el mejor diseño de productos, a partir de diferentes criterios cualitativos), también ha sido implementado con éxito en evaluación de tecnologías de la información a partir de la utilización de variables cualitativas (Zambrano, et al., 2010). En radio cognitiva, se usó la versión anterior a FAHP, que es AHP (Rodríguez, et al., 2011) y que fue tratado con anterioridad en este artículo. La ventaja prometedora es la manipulación de un número importante de métricas, sin incrementar la complejidad del sistema.

Inteligencia artificial (IA) (Dobrzański, et al., 2014). Se refiere a la manera artificial de plasmar el razonamiento propio del ser humano en dispositivos electrónicos volviéndolos autosuficientes en la toma de decisiones influenciados por la conducta que los rodea, con la propiedad de retroalimentarse así mismo para mejorar su probabilidad de éxito. Es utilizado en procesadores de texto, bases de datos distribuidas, en la electrónica en reconocimiento de patrones, búsqueda inteligente en Internet. Algunos de los tipos más conocidos de IA son las redes neuronales artificiales, lenguaje de máquina (ML) y máquinas de soporte vectorial (SVM) (Zhitao, et al., 2011).

ML (Lahouari, et al., 2014). Técnica que busca la creación de algoritmos capaces de aprender de las decisiones tomadas en el presente para mejorar su elección en el futuro. Una ventaja es su capacidad de aprendizaje autónomo. Desventajas: complejidad en su uso, costo computacional elevado que hace inviable su uso en redes Ad-Hoc.

\section{CONCLUSIONES}

Los Autores del presente artículo plantean la propuesta mostrada en la figura 12, con el fin de desarrollar un sistema predictivo de selección de canales de acuerdo con los requerimientos de calidad de servicio teniendo en cuenta no solamente el comportamiento de los usuarios primarios y sino también involucrando los secundarios, lo que permitirá un uso más equitativo y un aprovechamiento más adecuado de los medios, por parte de los nodos cognitivos que compongan la red CR. 
A partir de la situación actual en la toma de decisiones, es claro que existen varias temáticas que esperan ser abordadas y afianzadas en la CR como punto de apoyo para que el concepto de acceso dinámico en el espectro radioeléctrico pueda llegar a ser en un futuro valido comercialmente. Para ello varias temáticas deben ser investigadas:

El modelaje de la actividad del usuario secundario, variable que debe ser tenida en cuenta si se asume un crecimiento anticipado en el número de nodos en la red cognitiva. El monitoreo del secundario se justifica en la medida en que en muchos casos la cantidad de usuarios que acceden a las bandas es bastante alta, entonces es importante tener la posibilidad de estimar su comportamiento futuro en el canal para tener un control mucho más eficiente diseñando modelos confiables que estimen el comportamiento de los SU disminuyendo la probabilidad de colisiones entre SUs, y SUs - PUs.

El desarrollo de algoritmos inteligentes basados en autoaprendizaje para la selección espectral, utilizando una estrategia proactiva, disminuirían los tiempos necesarios para la asignación de bandas a los SUs optimizando el desempeño de la red.

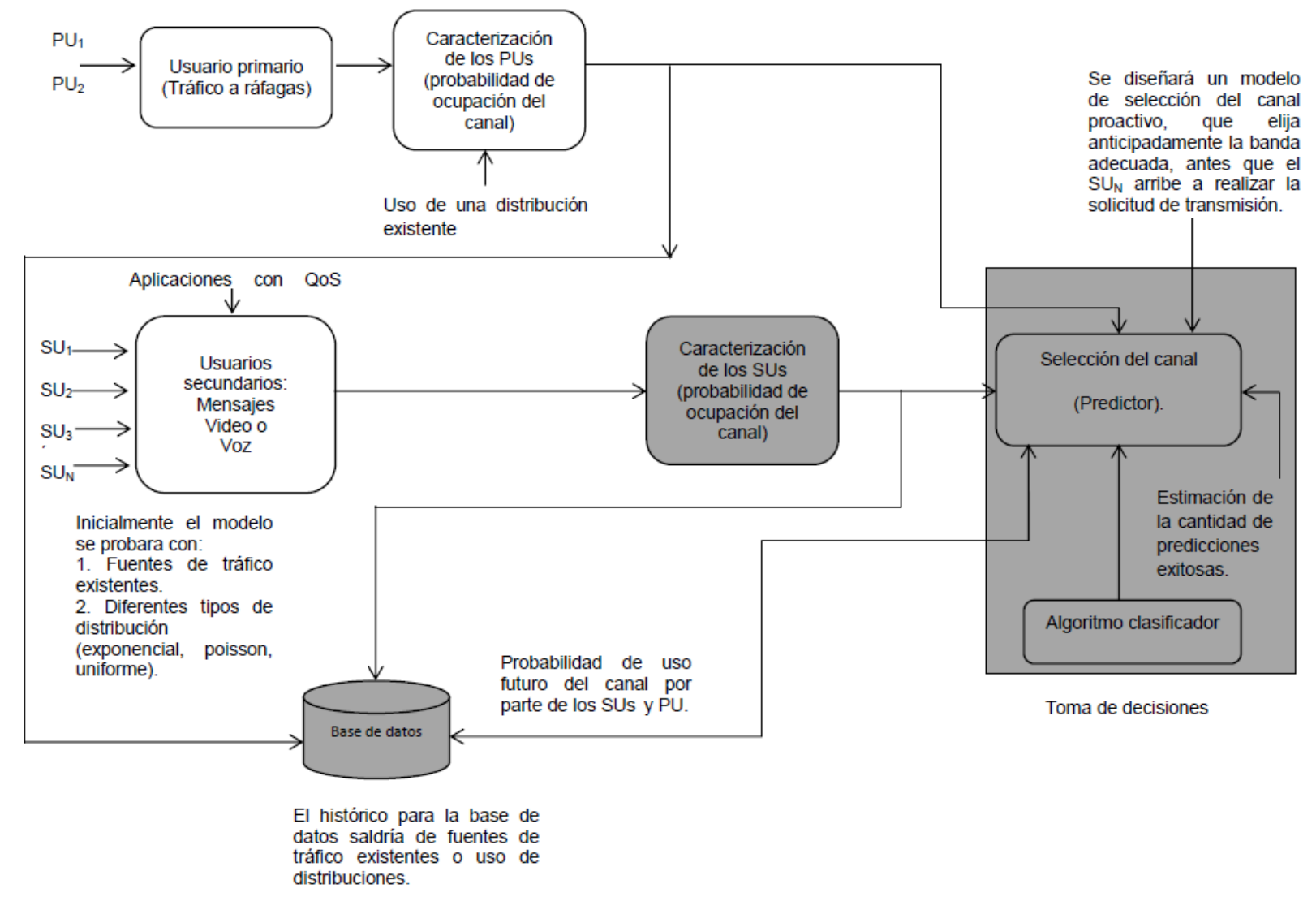

Fig. 12: Aproximación del modelo conceptual de la propuesta.

\section{REFERENCIAS}

Akyildiz, I., B. Lo, R. Balakrishnan, Cooperative spectrum sensing in cognitive radio networks: a survey, EISevier, Physical Communications, Volume 4, Issue 1, 40-62 (2011).

Almalfouh, S., G. Stber, Interference-aware radio resource allocation in OFDMA-based cognitive radio networks, IEEE Transactions Vehicular Technology, Volume 60, Issue 4, 1999-3004, May (2011).

Azarfar, A., J. Frigon., B. Sanso, Improving the reliability of wireless networks using cognitive radios, IEEE Communications Surveys Tutorials, Volume 14, Issue 2, 338-354 (2012).

Barnes, S., B. Maharaj, Prediction based channel allocation performance for cognitive radios, ElSevier, International Journal of Electronics and Communications, In Press (2013). 
Bolivar, N, Medium access control messaging scheme for cognitive radio networks, Tesis Doctoral, Departamento de Computación, Arquitectura y Tecnología, Universidad de Girona, España, Junio (2012).

Brah, F., I. Dayoub., L. Vandendorpe, Constrained resource allocation for OFDMA cognitive radio networks with primary users activity consideration, IEEE international symposium on wireless communication systems (ISWCS), 766-770 (2012).

Butun, I., A. Cagatay., D. Altilar., M. Khalid., R. Sankar, Impact of mobility prediction on the performance of Cognitive Radio networks, Wireless Telecommunications Symposium (WTS), Tampa FL, April 21-23 (2010).

Canberk, B., I. Akyildiz, S. Oktug, Primary user activity modeling using first-difference filter clustering and correlation in cognitive radio network, IEEE/ACM Transaction Networking, Volume 19, Issue 1, 170-183, February (2011).

Csurgai, L., J. Bito, Primary and secundary user activity models for cognitive wireless network, IEEE International Conference on Telecommunications (ConTEL), 301-306 (2011).

Derakhshani, M., T. Le-Ngoc, Learning-Based opportunistic spectrum access with adaptive hopping transmission strategy, IEEE Transactions on Wireless Communications, Volume 11, Issue 11, 3957-3967 (2012).

Dobrzański, L., Trzaska, J., Dobrzańska-Danikiewicz, A, Use of Neural Networks and Artificial Intelligence Tools for Modeling, Characterization, and Forecasting in Material Engineering, Comprehensive Materials Processing 2014, Volume 2: Materials Modeling and Characterization, 161-198 (2014).

Federal Communications Commission, Spectrum policy task force report, Tech. Rep. ET Docket 02-155, November (2002).

Ghosh, C., S. Pagadarai, D. Agrawal., A. Wyglinski, A framework for statistical wireless spectrum occupancy modeling, IEEE Transactions Wireless Communications, Volume 9, Issue 1, pp. 38-44, January (2010).

Gutiérrez, L., S. Zazo., J. Murillo., I. Álvarez., A. Garcia., B. Pérez, HF spectrum activity prediction model based on HMM for cognitive radio applications, EISevier, Physical Communications, Volume 9, 199-211 (2013).

Harris, P, Average net user online statistics, released in 2009, In Press, Corrected Proof Available online (2013).

Husain, M., A. Carneiro., H. Khalife., S. Fdida, SURF: A distributed channel selection strategy for data dissemination in multi-hop cognitive radio networks, EISevier, Computer Communications, Volume 36, Issue 10-11, 1172-1175 (2013)

IEEE 802.22, Working Group on Wireless Regional Area Networks, enabling Spectrum Sharing and Rural Broadband Wireless Access Using Cognitive Radio Technology in White Spaces, 2012. [En línea], consultado en Marzo 7 de 2014, disponible en: http://www.ieee802.org/22/.

IEEE Standard 802.22, Part 22: Cognitive wireless RAN medium access control (MAC) and Physical layer (PHY) specifications: Policies and procedures for operation in the TV bands, IEEE Computer Society, New York, USA, July (2011).

Khabazian, M., S. Aissa., N. Tadayon, Performance modeling of a two-tier primary- secondary network operated with IEEE802.11 DCF mechanism, IEEE Transaction Wireless Communications, 3047-3057 (2012).

Kolar, V., A. Gerco., T. Milcher., M. Petrova., P. Mahonen, Capacity estimation and adaptation in cognitive radio networks: Demonstrating software defined radios in action, International Conference on Mobile Computing and Networking (MobiCom), Beijing, 20-25, September (2009). 
Lahouari, G., Tarek, R., Sheltami, Khaled, S, Mobility Prediction in Mobile Ad Hoc Networks Using Extreme Learning Machines, The 4th International Conference on Ambient Systems, Networks and Technologies (ANT 2013), the 3rd International Conference on Sustainable Energy Information Technology (SEIT-2013), , Procedia Computer Science Volume 19, 305-312 (2013).

Lau, L., C. Lin., S. Wang, Bi-directional cognitive radio mac protocol for supporting TCP flows, IEEE Vehicular Technology Conference (VTC Fall), San Francisco (2011).

Liang, Z., S. Feng., D. Zhao., X. Shen, Delay performance analysis for supporting real-time traffic in a cognitive radio sensor network, IEEE Transaction Wireless Communications, Volume 10, Issue 1, 325-335, January (2011).

Lindberg, M, QoS Provisioning in Channel Allocation Strategy in Cognitive Radio Using Fuzzy Logic, American Journal of Electrical Systems, Volume 2, Issue 1, 11-22 (2012).

López, M., F. Casadevall., A. Umbert., J. Pérez-Romero., R. Hachemani., J. Palicot., C. Moy, Spectral occupation measurements and blind standard recognition sensor for cognitive radio networks, in International Conference Cognitive Radio Oriented Wireless Network Communications., Hannover, Germany, 1-9 (2009).

Masonta, M., M. Mzyece., N. Ntlatlapa, Spectrum decision in cognitive radio networks: A survey, IEEE Communications Society, Communications Surveys \& Tutorials, Volume 15, Issue 3, 1088-1107, July (2013).

Matinmikko, M., M. Hoyhtya., M. Mustonen., H. Sarvanko., A. Hekkala., M. Katz., A. Mammela., M. Kiviranta., A.. Kautio, Cognitive radio: an intelligent wireless communication system, Tech. Rep, (2008).

Matinmikko, M., M. Mustonen., M. Höyhtyä., T. Rauma., H. Sarvanko., A. Mämmelä, Distributed and directional spectrum occupancy measurements in the $2.4 \mathrm{GHz}$ ISM band, Symposium Wireless Communications, New York, United Kingdom, 976-980 (2010).

McHenry, M, NSF spectrum occupancy measurements project summary, Shared Spectrum Company, Tech. Rep, August (2005).

McHenry, M., P. A. Tenhula, D. McCloskey, D. A. Roberson, and C. S. Hood, Chicago spectrum occupancy measurements \& analysis and a long-term studies proposal, in Proc. Int. Workshop Technology Policy Accessing Spectrum, Boston, (2006).

Mitola, J, Software radios - survey, critical evaluation and future directions, in Proceedings of the National Telesystems Conference (NTC), Washington D.C, 13/15-13/23, May (1992).

Motani, M., Y. Liang, MAC protocol design and performance analysis for random access cognitive radio networks, IEEE Journal on Selected Areas In Communications, Volume 31, Issue 11, 2289- 2300 (2013).

Nie, N., C. Comaniciu, Adaptive channel allocation spectrum etiquette for cognitive radio networks, IEEE DySPAN, 269 -278, November (2005).

Ning, G., K. Chowdhury., J. Duan., P. Nintanavongsa, Licensed user activity estimation and track in mobile cognitive radio ad hoc networks, ElSevier, Computers and Electrical Engineering, 1705-1716 (2013).

Niyato, D., E. Hossain, Competitive pricing for spectrum sharing in cognitive radio networks: Dynamic game, inefficiency of Nash equilibrium, and collusion, IEEE Journal on Selected Areas in Communications (JSAC), Volume 26, Issue 1, $192-202$ (2008).

Park, J., D. Cho, QoS provisioning spectrum management based on intelligent matching and reservation for cognitive radio system, $2^{\text {nd }}$ International Conference on Cognitive Radio Oriented Wireless Networks and Communications, Orlando, 530-534 (2007).

Pla, V., J. Vidal., J. Martinez-Bauset., L. Guijarro, Modeling and characterization of spectrum white spaces for underlay cognitive radio networks, IEEE International Conference on Communications (ICC), Cape Town, South Africa, May 23-27 (2010). 
Rabbachin, A., T. Quek, H. Shin., M. Win, Cognitive network interference, IEEE Journal Selected Areas Communications, Volume 29, Issue 2, 480-493, February (2011).

Rodriguez, R., C. Ramirez., C. Carrillo, Multiple attribute dynamic spectrum decision making for cognitive radio networks, IEEE International Conference on Wireless and Optical Communications Networks (WOCN), Eighth International Conference France, 1-5 (2011).

Shukla, A., et al, Cognitive Radio Technology - A study for OFCOM, 2011. [En línea], consultado en Junio 10 de 2013, disponible en: http://enstakeholders.ofcom.org.uk/binaries/research/technologyresearch/ cograd main.pdf.

Song, C., Q. Zhang, Cooperative spectrum sensing with multi-channel coordination in cognitive radio networks, IEEE International Conference on Communications (ICC), Cape Town, May 23-27 (2010).

Suraweera, H., P. Smith., M. Shafi, Capacity limits and performance analysis of cognitive radio with imperfect channel knowledge, IEEE Transactions Vehicular Technology, Volume 59, Issue 4, 1811-1822, May (2010).

Suzan, B., A. Fatih, Distributed channel selection in CRAHNs: A non-selfish scheme for mitigating spectrum fragmentation, EISevier, Ad Hoc Networks, 774-788 (2012).

Taher, T., R. Bacchus., K. Zdunek., D. Roberson, Long-term spectral occupancy findings in Chicago, IEEE International Symposium Dynamic Spectrum. Access Networks, Germany, 100-107 (2011).

Timmers, M., S. Pollin., A. Dejonghe., L. Van., F. Catthoor, A Distributed Multichannel MAC Protocol for Multihop Cognitive Radio Networks, IEEE Transactions on Vehicular Technology, Volume 59, Issue 1 (2011).

Vishram, M., C. Lau., Ch. Syin., K. Ashish, Energy aware spectrum decision framework for cognitive radio networks, Electronic System Design (ISED), IEEE International Symposium, Kolkata, December 19-22 (2012).

Vishram, M., C. Lau., S. Chan, QoS based spectrum decision framework for cognitive radio networks, Networks (ICON), IEEE International Conference on IEEE, 18-23, Singapore (2012).

Wang, J., M. Ghosh, K. Challapali, Emerging cognitive radio applications: a survey, IEEE Communications Magazine, Volume 49, Issue 3, 74-81, March (2011).

Wellens, M., A. de Baynast., P. Mähönen, Performance of dynamic spectrum access based on spectrum occupancy statistics, IET Communications, Volume. 2, Issue 6, 772-782, July (2008).

Wellens, M., J. Wu, P. Mähönen, Lessons learned from an extensive occupancy measurement campaign and stochastic duty cycle, Mobile Network, Volume 15, Issue 3, 461-474, January (2010).

Won-Yeol L., I. Akyildiz, Optimal spectrum sensing framework for cognitive radio networks, IEEE Transaction Wireless Communications, Volume 7, Issue 10, 3845-3857 (2008).

Won-Yeol, L., I. Akyildiz, Fellow, A spectrum decision framework for cognitive radio networks, IEEE Transactions on Mobile Computing, Volume 10, Issue 2, 161-174 (2011).

Won-Yeol, L., I. Akyldiz, A spectrum decision framework for cognitive radio networks, IEEE Transactions on Mobile Computing, Volume 10, Issue 2, 161-174 (2011).

Xiaoshuang, X., J. Tao., C. Wei., H. Yan., C. Xiuzhen, Spectrum prediction in cognitive radio networks, IEEE Wireless Communications, Volume 20, Issue 2, 90-96 (2013).

Xing, X., et al, channel quality prediction based on Bayesian Inference in Cognitive Radio Networks, IEEE INFOCOM, 2013.

Xing, X., T. Jing., W. Cheng., Y. Huo., X. Cheng, Spectrum prediction in cognitive radio networks, IEEE Wireless Communications, Volume 20, Issue 2, 90-96 (2013).

Xu, D., E. Jung., X. Liu, Optimal bandwidth selection in multichannel cognitive radio networks: How much is too much?, IEEE Int. Symposium for New Frontiers in DySPAN, Illinois, USA, October 14-17 (2008). 
Yarkan, S., H. Arslan, Binary time series approach to spectrum prediction for cognitive radio, IEEE Vehicular Technology Conference, Baltimore, USA, September/October 30-3 (2007).

Yuan, Y, P. Bahl., R. Chandra., T. Moscibroda, Y. Wu, Allocating dynamic time-spectrum blocks in cognitive radio networks, ACM International Symposium on Mobile ad hoc Networking and Computing Wireless Communications and Networking Conference, Quebec, Canada, September 9-14 (2007).

Zambrano, J., M. Valencia., C. Solórzano, Software para seleccionar el mejor diseño de producto con múltiples criterios cualitativos y con un equipo de diseño multidisciplinar, IEEE Concapan, Ponencia en Costa Rica (2010).

Zhitao, Z., W. Lifeng., D. Guoru., W. Shengqun, Enhanced spectrum decision based on the combination of sensing and prediction, Cross Strait Quad-Regional Radio Science and Wireless Technology Conference (CSQRWC), Volume 2, 985 -989, July (2011). 